\title{
Neurogenic Dyspnea: Is Idiopathic Dyspnea Related to a Post-Viral Sensory Neuropathy? A Case Series Supporting a New Hypothesis
}

\author{
Zalvan $\mathrm{C}^{1,2,3 *}$, Yuen E', Cole J ${ }^{4}$, Loftus $\mathrm{C}^{4}$, Geliebter \\ $\mathrm{J}^{1,5}$ and Nguyen $\mathrm{SA}^{6}$ \\ ${ }^{1}$ New York Medical College, School of Medicine, USA \\ ${ }^{2}$ Institute for Voice and Swallowing Disorders, Phelps \\ Hospital, USA \\ ${ }^{3}$ ENT and Allergy Associates, LLP, USA \\ ${ }^{4}$ Department of Otolaryngology, University of Rochester, \\ USA \\ ${ }^{5}$ Department of Microbiology and Immunology, New York \\ Medical College, USA \\ ${ }^{6}$ Department of Otolaryngology, Medical University of \\ South Carolina, USA \\ *Corresponding author: Zalvan C, Institute for Voice \\ and Swallowing Disorders, Phelps Hospital, Northwell \\ Health, ENT and Allergy Associates, LLP, $358 \mathrm{~N}$ \\ Broadway Suite 203, Sleepy Hollow, NY 10591, USA
}

Received: January 18, 2021; Accepted: February 02, 2021; Published: February 09, 2021

\begin{abstract}
Objective: To propose a hypothesis of a novel potentially post-viral sensory neuropathic disorder, termed neurogenic dyspnea, and assess the therapeutic efficacy of a trigger reduction approach in this unique patient population.
\end{abstract}

Methods: A retrospective chart review of patients seen between January 2011 and April 2018 for persistent dyspnea of unknown etiology was conducted. All patients failed to improve with treatment for presumptive diagnoses of allergy, asthma, sinus disease, and reflux. Patients were educated on our treatment protocol consisting of a Mediterranean style, plant-based diet with alkaline water, saline irrigation, and combined azelastine/fluticasone nasal spray. Treatment response was followed using two validated symptom questionnaires, Reflux Symptom Index (RSI) and Dyspnea Index (DI).

Results: Of 57 initial patients, 8 were included in the final analysis. Mean age was 38.8 years (range 11-68). Six (75\%) patients were female. All patients were followed for a minimum of 4 weeks. Using the reduction in DI and RSI as continuous variables to assess response, patients experienced a $9.4(95 \% \mathrm{Cl}$ : 3.9-14.9) and 14.1 (95\% Cl: 6.0-22.2) mean point reduction, respectively. One patient saw a $100 \%$ and another a $95 \%$ reduction in DI. Six of eight patients experienced more than a $50 \%$ reduction in $\mathrm{RSI}$.

Conclusion: We hypothesize that Neurogenic Dyspnea is a newly described clinical phenomenon with a possible post-viral etiology. This pilot study demonstrated that our trigger reduction approach improved subjective symptoms in the majority of patients with unexplained dyspnea with previous treatment failure for common etiologies. Further studies are required to validate these findings.

Keywords: Virus diseases; Dyspnea; Laryngopharyngeal reflux; Therapeutic irrigation

\section{Abbreviations}

ASIC: Acid Sensing Ion Channel; CI: Confidence Index; DI: Dyspnea Index; LPR, Laryngopharyngeal Reflux; ND: Neurogenic Dyspnea; PFT: Pulmonary Function Test; RAR: Rapidly Adapting Receptors; RSI: Reflux Severity Index; SAR: Slowly Adapting Receptors; SARS-Cov-2: Severe Acute Respiratory Syndrome Coronavirus-2; TRP: Transient Receptor Potential; URI: Upper Respiratory Infection

\section{Introduction}

Dyspnea is a subjective feeling of breathlessness that leads to undue anxiety and stress. It is a common finding in individuals suffering from various forms of known laryngeal and pulmonary dysfunction [1,2]. Interestingly, we have observed dyspnea in patients with no organic diagnosis that would otherwise explain these symptoms. Many of these patients report the sensation of "air hunger," or the inability to obtain a full breath, and are incorrectly diagnosed with chronic asthma despite normal pulmonary function testing and imaging, and little to no response to typical asthma pharmacotherapy. Recent analysis of asthma patients suggests upward of 30\% of these patients are incorrectly diagnosed with asthma and nearly $90 \%$ are able to discontinue their asthma medication use [3].

We further present our hypothesis that a potentially post-viral change to the afferent sensory arm of the respiratory tract can lead to altered sensory feedback and result in a feeling of shortness of breath in the absence of true objective respiratory compromise. To our knowledge, this is the first case series of patients with dyspnea not related to a readily identifiable underlying organic cardiopulmonary pathology or anatomical abnormality. Modelled after a trigger reduction treatment regimen for chronic neurogenic cough [4] and paradoxical vocal fold motion [5], we employed a therapeutic trigger reduction protocol including a 90-95\% plant-based, Mediterranean style diet with alkaline water to decrease Laryngopharyngeal Reflux (LPR) [6], saline irrigation, and combined azelastine/fluticasone nasal spray for basal post-nasal drip reduction. Theoretically decreasing the basal rate of nasal drainage and physiologic reflux results in less stimulation of the laryngopharyngeal tissues with less afferent stimulation. Additional treatments such as respiratory
Austin J Otolaryngol - Volume 8 Issue 1 - 2021

ISSN : 2473-0645 | www.austinpublishinggroup.com

Zalvan et al. (C) All rights are reserved
Citation: Zalvan C, Yuen E, Cole J, Loftus C,Geliebter J and Nguyen SA. Neurogenic Dyspnea: Is Idiopathic Dyspnea Related to a Post-Viral Sensory Neuropathy? A Case Series Supporting a New Hypothesis. Austin J Otolaryngol. 2021; 8(1): 1119. 
retraining therapy [7] and/or neuromodulatory pharmacotherapy [8] in patients who fail to respond will be reviewed.

\section{Materials and Methods}

This is a New York Medical College IRB approved retrospective chart review conducted between January 2011 and April 2018 using the following ICD-9 and ICD-10 codes: R05.0, 786.05, 519.9, 519.8, 784.99, 786.09, 786.9, R06.02, J98.9, J98.8, R06.89 and R06.09. Charts were reviewed and information was recorded on demographics, history of present illness, medical and surgical history, medications, allergies, pulmonary function test (PFT) results, and treatment regimen. Laryngoscopic examination was performed to rule out laryngeal pathology such as vocal paralysis, subglottic stenosis and other laryngeal lesions. Response to treatment was gauged by a change in the validated indices, Reflux Symptom Index (RSI) [9] to assess changes in laryngeal symptoms, both reflux related and other non-specific laryngopharyngeal symptoms, and Dyspnea Index (DI) [10], to quantify the severity of symptoms in upper airway dyspnea. A 6-point or greater reduction in RSI [11] and a decrease of 4 or more for DI [12] were considered clinically meaningful. Inclusion criteria were a documented history of persistent, baseline dyspnea, DI and RSI documented on initial presentation and minimum 4-week followup, and a documented treatment regimen. Additionally, patients demonstrated prior failure to improve with empiric treatments for allergy, asthma, reflux and sinus disease even in the setting of normal objective findings for these disease states. Exclusion criteria included confirmed diagnoses of asthma, chronic obstructive pulmonary disease, congestive heart failure, bronchiectasis, restrictive lung disease, active pulmonary infection, stenotic disease of the upper airway, presence of lesions causing mass effect on the upper airway, smoking history, charts lacking documented DI and RSI, and/or abnormal PFT results. Some patients report a preceding Upper Respiratory Tract Infection (URTI) prior to symptom onset.

Patients were educated and provided guidelines on our nonsystemic, trigger reduction approach for management of dyspnea of unknown etiology. Intervention consisted of a Mediterranean style, 90-95 \% plant-based diet for treatment of LPR: three to four meals weekly with 2-3 oz of any type of meat or dairy, alkaline water, and standard reflux precautions including significant reduction of coffee, tea, chocolate, soda, greasy or fried food, fatty food, spicy food, alcohol, or acidic foods, especially if they are specific triggers. In addition, patients were instructed to remain upright for 3 hours prior to sleep, eat smaller and more frequent meals, elevate head of bed, as well as practice sinus toilet consisting of nasal saline and a nasal steroid/antihistamine twice daily. Patients were instructed to spray an aerosolized saline in each nostril for 3 seconds while sniffing inward forcefully until the saline entered the nasopharynx and was then expectorated. They were instructed to repeat this maneuver five times per day. Compliance was assessed historically with patient reporting fewer than three meals per week with 3-4 oz of any animal product.

Statistical analysis was performed in Microsoft Excel 2016. Patients were not stratified by treatment regimen as sample size was not large enough to calculate the effect of individual treatment regimens on outcomes. 95\% Confidence Intervals (CI) for the mean reduction in DI and RSI were determined. All statistics were calculated at $\alpha=0.05$.

\section{Results}

57 patients met initial criteria, 49 of which were excluded due to medical comorbidity or incomplete chart documentation. Only the eight patients who met criteria with documented pre- and posttreatment DI and RSI, and a documented trigger reduction treatment regimen were included in this analysis. Patient demographics are displayed in (Table 1). Patient age range was 11-68 years (mean $=38.8$ years) and mean time to follow-up was 7.8 weeks. There were six females and two males. The mean DI and RSI on initial presentation were 21.1 and 24.1, respectively. The mean DI and RSI scores at followup were 11.8 and 10, respectively. Using the reduction in DI and RSI after treatment as continuous variables to assess response, patients experienced a 9.4 (95\% CI: 3.9-14.9) and 14.1 (95\% CI: 6.0-22.2) mean point reduction, respectively. The mean percent reductions in DI and RSI were 36.0\% (95\% CI: 5.7-66.3) and 59.2\% (95\% CI: 35.4-83.0), respectively. One patient saw a complete (i.e. 100\%) and another saw a $95 \%$ reduction in DI. Six of the eight patients experienced more than a $50 \%$ reduction in RSI.

In total, seven of eight and seven of eight patients saw an absolute reduction in RSI and DI, respectively (Figures 1-3). Of the seven who saw a reduction in RSI, six had a reduction of at least six points. Of the seven who had a reduction in DI, they all decreased by at least four points. All eight patients received basic reflux trigger reduction

Table 1: Patient Demographics.

\begin{tabular}{|c|c|c|c|c|c|c|c|c|c|c|c|c|c|c|c|}
\hline \multicolumn{4}{|c|}{ Characteristics } & \multicolumn{3}{|c|}{ Past Medical History } & \multicolumn{4}{|c|}{ Symptoms } & \multicolumn{2}{|c|}{ Outcomes } & \multicolumn{3}{|c|}{ Therapies } \\
\hline \# & Sex & Age & BMI & Anxiety/Panic & Allergies & URI & Cough & Globus & Dysphagia & $\begin{array}{c}\geq 6 \\
\text { months }\end{array}$ & $\begin{array}{c}\text { DI } \\
\text { Reduction }\end{array}$ & $\begin{array}{c}\text { RSI } \\
\text { Reduction }\end{array}$ & PBD & Amitriptyline & RRT \\
\hline 1 & M & 41 & 25 & & $\mathrm{x}$ & $\mathrm{x}$ & $\mathrm{x}$ & & & & 19 & 34 & $x$ & & \\
\hline 2 & $\mathrm{~F}$ & 52 & 25 & & & $\mathrm{x}$ & & $\mathrm{x}$ & & & -2 & 8 & $\mathrm{x}$ & & \\
\hline 3 & $\mathrm{~F}$ & 11 & 15 & $x$ & $x$ & & & $\mathrm{x}$ & $x$ & $\mathrm{x}$ & 13 & 13 & $\mathrm{x}$ & & $\mathrm{x}$ \\
\hline 4 & $\mathrm{~F}$ & 68 & 18 & $x$ & $x$ & & $\mathrm{x}$ & & $x$ & $x$ & 21 & 26 & $x$ & & \\
\hline 5 & $\mathrm{~F}$ & 65 & 36 & & & & $x$ & & $x$ & $\mathrm{x}$ & 5 & 0 & $x$ & & \\
\hline 6 & $\mathrm{~F}$ & 34 & 24 & $x$ & $\mathrm{x}$ & & & & $\mathrm{x}$ & $\mathrm{x}$ & 10 & 19 & $x$ & $x$ & $\mathrm{x}$ \\
\hline 7 & $\mathrm{~F}$ & 23 & 20 & & & & $x$ & & & & 5 & 11 & $\mathrm{x}$ & & \\
\hline 8 & M & 16 & 18 & & $\mathrm{x}$ & $x$ & & $\mathrm{x}$ & & $\mathrm{x}$ & 4 & 2 & $\mathrm{x}$ & & \\
\hline \multicolumn{4}{|c|}{ Totals $(n=8)$} & 3 & 5 & 3 & 4 & 3 & 4 & 5 & & & 8 & 1 & 2 \\
\hline
\end{tabular}

Abbreviations: BMI: Body Mass Index; URI: Upper Respiratory Tract Infection; DI: Dyspnea Index; RSI: Reflux Severity Index; PBD: Plant-Based Diet; RRT: Respiratory Retraining Therapy 


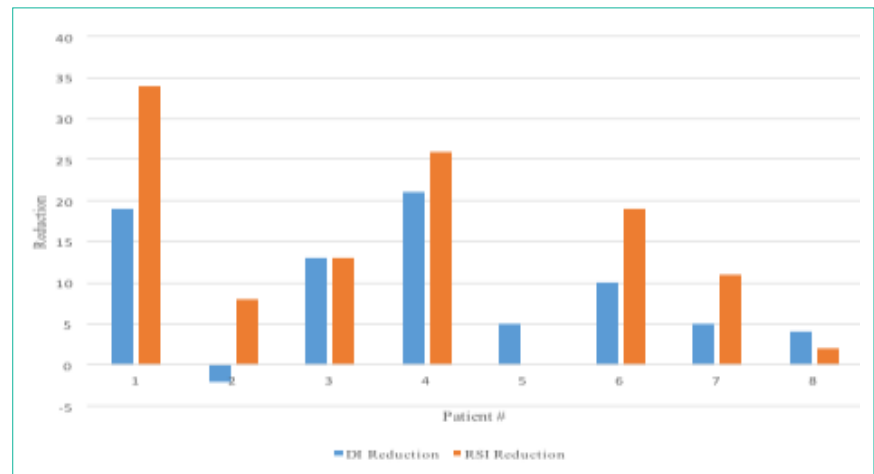

Figure 1: Change in Dyspnea Index (DI) and Reflux Severity Index (RSI) Following Treatment Protocol in Our 8-Patient Cohort.

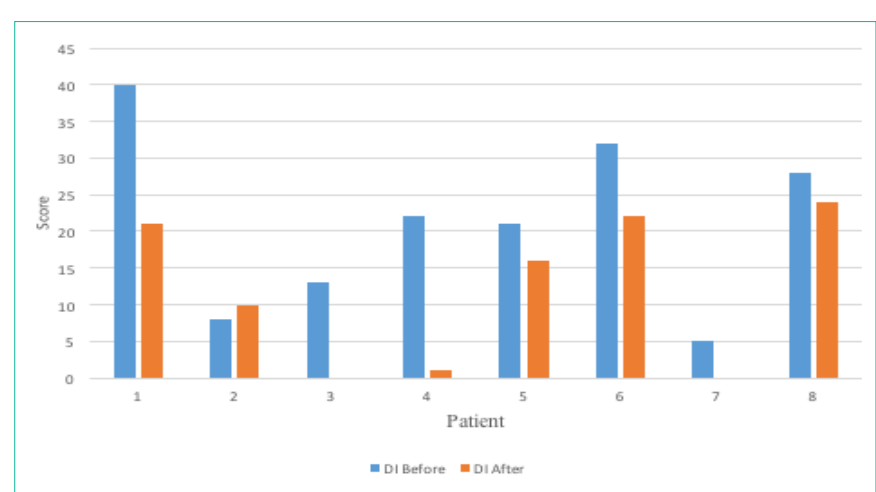

Figure 2: Pre- and Post-Treatment Dyspnea Index (DI) Scores in Our 8-Patient Cohort.

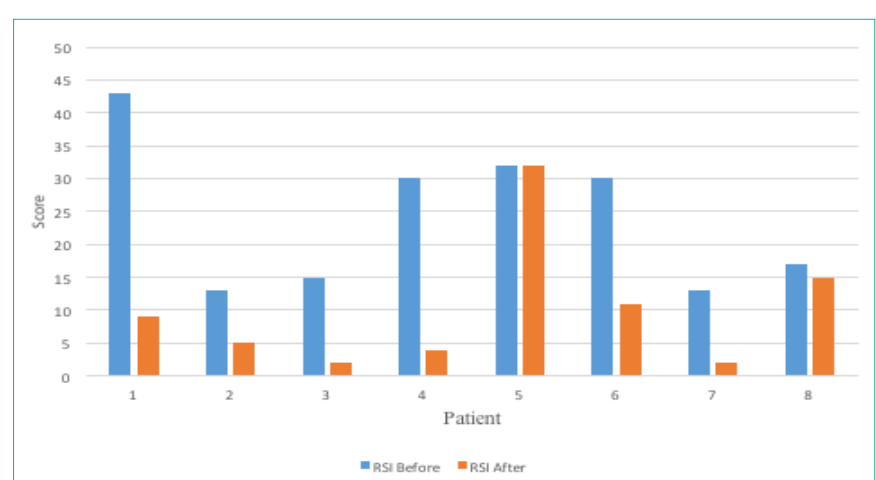

Figure 3: Pre- and Post-Treatment Reflux Severity Index (RSI) Scores in Our 8-Patient Cohort.

therapy and azelastine/fluticasone nasal spray. All patients were compliant with a $95 \%$ plant-based diet with alkaline water, one patient was placed on amitriptyline after failure of response, and two patients underwent concurrent respiratory retraining therapy. Of the eight patients placed on the $95 \%$ plant-based diet with alkaline water, six had a concomitant reduction in both DI and RSI. One patient saw no change in RSI. One patient was observed to have a slight increase (2 points) in DI.

\section{Discussion}

Dyspnea is a subjective feeling of breathlessness often resulting in considerable anxiety and stress. Interestingly, we have observed dyspnea as an isolated complaint in patients with no organic diagnosis that would otherwise explain this symptom. Although the neural mechanisms that contribute to dyspnea remain elusive, there is growing evidence that central, peripheral, and chemoreceptor mechanisms are involved. Numerous types of vagal afferent receptors have been described in the respiratory tract, some of which have been linked to the pathophysiology of dyspnea, namely Slowly Adapting Receptors (SARs), rapidly adapting receptors (RARs), and unmyelinated C-fibers [13]. Airway inflammation can occur from allergy, post-nasal drainage from sinus inflammation, laryngopharyngeal reflux, and asthma. Stimulation of airway afferents from these processes can result in activation of parasympathetic vagal efferents causing smooth muscle contraction and increased airway resistance. This can lead to the sensation of dyspnea. Interestingly, independent of smooth muscle contraction, the same inflammation can lead to increased afferent activity, especially of the c-fibers or mechanoreceptors. This afferent activity results in the sensation of dyspnea without any physical change in the airway caliber [14].

The authors will henceforth refer to this phenomenon as Neurogenic Dyspnea (ND), defined as a sensation of shortness of breath in the absence of any physical and measurable correlate. As with chronic neurogenic cough, post-viral anosmia, dysgeusia and the spectrum of vocal paresis, ND is potentially another manifestation of a post-viral sensory neuropathic disorder. As such, symptoms of ND are similar to the spectrum of post-viral neuropathic symptoms chronic cough, globus sensation, burning and pain syndromes of the pharynx, and vocal cord dysfunction.Often observed, patients will typically take a prolonged inspiration with chest expansion followed by abrupt exhalation, often while shrugging shoulders upward repeatedly throughout the evaluation. This "dyspneic gasp" is often reported by relatives who witness this maneuver throughout the day but do not observe it while the patient is asleep.

Recent reports of viral-associated olfactory loss secondary to Severe Acute Respiratory Syndrome Coronavirus 2 (SARS-CoV-2) infection demonstrate yet another post-viral neuropathic sensory deficit $[15,16]$. It is indeed intriguing to consider ND as a potential etiology of the breathlessness and shortness of breath in many of the post-COVID "long-haulers" who present without physical explanation for their symptoms $[17,18]$.

In our eight-patientcohort, two subjects were previously diagnosed with asthma by another provider. Only one was receiving asthma pharmacotherapy, with no relief of her dyspnea, despite normal PFT findings at initial presentation with no evidence of bronchial hypersensitivity during functional testing. Aaron et al. demonstrated that a third of patients with physician-diagnosed asthma were misdiagnosed and subsequently weaned off their medications [3]. Review of our entire population demonstrated similar rates of normal PFT findings of approximately 30\% (data not published). Our cohort of patients in this initial pilot study and definition of the disease, all patients had normal PFT. As such, in the absence of clinical or laboratory evidence of asthma, we propose that $\mathrm{ND}$ may be responsible for some of the unexplained perception of shortness of breath in a subset of these patients.

Symptom presentation is often skewed by underlying psychiatric influence. Dyspnea, especially in this ND population, is often treated for months or years without significant relief adding to the 
anxiety. Underlying mood disorders can heighten dyspnea with depression and anxiety commonly being present in many respiratory diseases [17], often with normal pulmonary function [18]. The central processing of respiratory symptoms can be modified by emotionswith the anterior cingulate cortex and insula regulating the sensory response to respiratory stimuli. These two brain regions may be hyperresponsive in those with stress-related conditions through unclear neural mechanisms [19]. In our cohort with suspected ND, only three of eight reported having an anxiety disorder, possibly from underreporting or because a mood disorder was not the primary etiology driving this multifactorial disease process. Nonetheless, psychotherapy, in conjunction with other modalities, may be beneficial to select patients with a known or suspected mood disorder contributing to their perceived shortness of breath. Furthermore, there is evidence that gender affects the perception of dyspnea, with women suffering more strongly from dyspnea than men, likely from sex hormone differences [20]. Our data concurs with this observed gender disparity, as $75 \%$ of our cohort were females.

In the absence of laryngeal and cardiopulmonary abnormalities following an extensive diagnostic workup, initial treatment for $\mathrm{ND}$ is warranted with a trigger reduction approach similar to that used in the management of neurogenic chronic cough [4]. Our approach utilizes a plant-based Mediterranean-style diet combined with alkaline water and standard reflux precautions for LPR and sinus toilet consisting of nasal saline irrigation and a nasal steroid/ antihistamine combination for postnasal drainage reduction. The peripheral neuronal hyperexcitability of the tracheobronchial tree is mediated by a combination of peripheral and central sensitization via upregulation of a host of receptors implicated in the pathogenesis of dyspnea. Transient Receptor Potential (TRP) ion channels, notably TRPV1 and Acid-Sensing Ion Channels (ASIC) represent two such types of receptors expressed on vagal afferent C-fibers that innervate the respiratory tract and are activated by a decrease in the extracellular $\mathrm{pH}$ [21], such as in the case with LPR. Additionally, increases in carbon dioxide, as seen with increased activity, can result in localized reduction of $\mathrm{pH}$, especially in the setting of inflammation [22]. To theoretically dampen the peripheral stimulation triggered by postnasal drainage, our regimen can potentially thin out and decrease the total amount of mucus, with its mix of cytokines and inflammatory mediators, draining into the nasopharynx. This approach may decrease the stimulation of various mechano and chemoreceptors implicated in the pathogenesis of dyspnea throughout the respiratory tract [5]. Furthermore, exposing human bronchial epithelium to a variety of respiratory viruses upregulates ASIC and TRPV1 expression, potentially contributing to the post-viral hypersensitivity often observed in dyspneic patients with no identifiable etiology [23]. In our cohort, three patients reported having an URI just prior to the onset of their shortness of breath. Three patients reportedly did not experience an URI while two were unsure. Although our study weakly supports a post-viral vagal neuropathy as the underlying cause, it is possible that the other $62.5 \%$ either had a subclinical presentation of a viral URI or were unable to recall having had an URI, reflecting an inherent problem of this retrospective study. Recall of URI symptoms are often similar in patient populations presenting with acute sensorineural hearing loss, Bell's palsy, and acute vocal fold paresis. Increased expression results in increased sensitivity of the airway afferents. Increases in peripheral afferent neuronal activity secondary to hypersensitivity can result in synaptic augmentation resulting in central sensitization resulting in increased cough and the sensation of dyspnea [24]. Trigger reduction, reducing inflammatory mediators, can result in potential alleviation of these symptoms.

Seven of our eight patients reported improvement in the severity of their laryngopharyngeal and reflux symptoms, as evidenced by an absolute reduction in RSI following our trigger reduction approach. Of these seven subjects, six experienced a clinically significant response, as suggested by a reduction of at least six points. Since the subjective sensation of shortness of breath was the most distressing complaint in this cohort with suspected ND, our therapeutic protocol aimed to achieve at least a four-point change in DI to effect a clinically meaningful response as the primary outcome. Of eight patients, sevenachieved this outcome, reporting improvement in the severity of their dyspnea post-treatment. For patients who fail trigger reduction alone, respiratory retraining therapy can be an effective adjunct, as positive results have been observed in patients treated for paradoxical vocal fold motion [25] and neurogenic cough [26]. Psychotherapy may be beneficial for those with comorbid disorders. For dyspnea refractory to trigger reduction for six weeks followed by respiratory retraining therapy, pharmacotherapy can start with a trial of amitriptyline. Gabapentin is typically used first in the older population due to its fewer side effects and drug interactions. If these medications fail, tramadol followed by pregabalin can be considered [27].

There are several inherent biases and limitations in this pilot retrospective study. The primary limitation of this study is the low number of subjects evaluated. Additionally, reflux testing was not performed. Although all patients were instructed to practice standard reflux precautions, whether full compliance with our plant-based, Mediterranean-style LPR diet was achieved is uncertain. We also acknowledge that our treatment approach consisting of sinus toilet and diet may exert a placebo effect that can influence the patients' response to the subjective questionnaires, whose validity has been questioned in the literature [28]. Furthermore, without a control group, it is unknown whether our therapeutic protocol effected a change in patient symptoms. Randomized controlled trials with a larger sample size are required to support the results of this study and further confirm our hypothesis.

\section{Conclusion}

Similar to the proposed pathophysiology of laryngeal dysfunction where irritants, inflammation, or infection may lead to sensitization of neuronal reflexes, we postulate that this observed phenomenon is also neurogenic in etiology and is perhaps a form of vagal neuropathy that results from the sensitization of neuronal receptors responsible for respiratory drive. Neurogenic dyspnea represents another potential post-viral sensory neuropathy affecting the upper aerodigestive tract. In patients with a subjective sensation of dyspnea in the absence of physical findings, a normal pulmonary workup, or poor treatment response for asthma, ND should be considered. Morbidity, cost, and anxiety associated with chronic asthma treatment can be avoided. Treatment with a trigger reduction approach using a $95 \%$ plant-based Mediterranean style diet and alkaline water to treat LPR combined with reduction of the basal rate of nasal drainage using saline irrigation, intranasal steroids, and antihistamine can improve 
subjective symptoms in the majority of patients presenting with this condition.

\section{References}

1. Hayen A, Herigstad M, Pattinson KT. Understanding dyspnea as a complex individual experience. Maturitas. 2013; 76: 45-50

2. Cukier-Blaj S, Bewley A, Aviv JE, Murry T. Paradoxical vocal fold motion: a sensory-motor laryngeal disorder. Laryngoscope. 2008; 118: 367-370.

3. Aaron SD, Vandemheen KL, FitzGerald JM, Ainslie M, Gupta S, Lemicre C et al. Reevaluation of Diagnosis in Adults with Physician-Diagnosed Asthma. Jama. 2017; 317: 269-279.

4. Zalvan C, Berzofsky C, Tiwari R, Geliebter J. Trigger Reduction Prior to Systemic Drugs for Neurogenic Chronic Cough. Laryngoscope. 2019; 129 : 198-202.

5. Zalvan C, Yuen E, Geliebter J, Tiwari R. A Trigger Reduction Approach to Treatment of Paradoxical Vocal Fold Motion Disorder in the Pediatric Population. J Voice. 2019.

6. Zalvan $\mathrm{CH}$, Hu S, Greenberg B, Geliebter J. A Comparison of Alkaline Water and Mediterranean Diet vs Proton Pump Inhibition for Treatment of Laryngopharyngeal Reflux. JAMA Otolaryngol Head Neck Surg. 2017; 143 : 1023-1029.

7. Nacci A, Fattori B, Segnini G, Dallan I, Panicucci E, Rocchi V, et al Respiratory retraining therapy in long-term treatment of paradoxical vocal fold dysfunction. Folia Phoniatr Logop. 2011; 63: 134-141.

8. Vertigan AE, Kapela SL, Ryan NM, Birring SS, McElduff P, Gibson PG Pregabalin and Speech Pathology Combination Therapy for Refractory Chronic Cough: A Randomized Controlled Trial. Chest. 2016; 149: 639-648.

9. Belafsky PC, Postma GN, Koufman JA. Validity and reliability of the Reflux Symptom Index (RSI). J Voice. 2002; 16: 274-277.

10. Gartner-Schmidt JL, Shembel AC, Zullo TG, Rosen CA. Development and validation of the Dyspnea Index (DI): a severity index for upper airway-related dyspnea. J Voice. 2014; 28: 775-782.

11. Lien HC, Wang CC, Lee SW, Hsu JY, Yeh HZ, Ko CW, et al. Responder Definition of a Patient-Reported Outcome Instrument for Laryngopharyngea Reflux Based on the US FDA Guidance. Value Health. 2015; 18: 396-403.

12. Gray AJ, Huston M, Didericksen D, Meyer TK, Merati A, Brisebois S. The minimal clinically important difference of the dyspnea index in laryngotracheal stenosis. Laryngoscope. 2020; 130: 1775-1779.

13. Canning BJ, Chou YL. Cough sensors. I. Physiological and pharmacological properties of the afferent nerves regulating cough. Handb Exp Pharmacol. 2009: $23-47$

14. Lee LY. Respiratory sensations evoked by activation of bronchopulmonary C-fibers. Respir Physiol Neurobiol. 2009; 167: 26-35
15. Soler ZM, Patel ZM, Turner JH, Holbrook EH. A primer on viral-associated olfactory loss in the era of COVID-19. International Forum of Allergy \& Rhinology. 2020; 10: 814-820.

16. Brann DH, Tsukahara T, Weinreb C, Lipovsek M, den Berge KV, Gong B, et al. Non-neuronal expression of SARS-CoV-2 entry genes in the olfactory system suggests mechanisms underlying COVID-19-associated anosmia. Sci Adv. 2020; 6: eabc5801.

17. Bogaerts K, Notebaert K, Van Diest I, Devriese S, De Peuter S, Van den Bergh $\mathrm{O}$. Accuracy of respiratory symptom perception in different affective contexts. J Psychosom Res. 2005; 58: 537-543.

18. von Leupoldt A, Chan PY, Bradley MM, Lang PJ, Davenport PW. The impact of anxiety on the neural processing of respiratory sensations. Neuroimage. 2011; 55: 247-252.

19. Rosenkranz MA, Davidson RJ. Affective neural circuitry and mind-body influences in asthma. Neuroimage. 2009; 47: 972-980.

20. Nowobilski R, Furgal M, Polczyk R, de Barbaro B, Szczeklik A. Gender gap in psychogenic factors may affect perception of asthma symptoms. J Investig Allergol Clin Immunol. 2011; 21: 193-198.

21. Undem BJ, Nassenstein C. Airway nerves and dyspnea associated with inflammatory airway disease. Respir Physiol Neurobiol. 2009; 167: 36-44.

22. Lin RL, Gu Q, Lin YS, Lee LY. Stimulatory effect of $\mathrm{CO} 2$ on vagal bronchopulmonary C-fiber afferents during airway inflammation. J Appl Physiol (1985). 2005; 99: 1704-1711.

23. Omar S, Clarke R, Abdullah $\mathrm{H}$, Brady C, Corry J, Winter $\mathrm{H}$, et al. Respiratory virus infection up-regulates TRPV1, TRPA1 and ASICS3 receptors on airway cells. PLoS One. 2017; 12: e0171681.

24. Mazzone SB, Undem BJ. Vagal Afferent Innervation of the Airways in Health and Disease. Physiol Rev. 2016; 96: 975-1024.

25. Marcinow AM, Thompson J, Forrest LA, deSilva BW. Irritant-Induced Paradoxical Vocal Fold Motion Disorder: Diagnosis and Management. Otolaryngol Head Neck Surg. 2015; 153: 996-1000.

26. Soni RS, Ebersole B, Jamal N. Treatment of Chronic Cough. Otolaryngo Head Neck Surg. 2017; 156: 103-108.

27. Greene SM, Simpson CB. Evidence for sensory neuropathy and pharmacologic management. Otolaryngol Clin North Am. 2010; 43: 67-72.

28. Park KH, Choi SM, Kwon SU, Yoon SW, Kim SU. Diagnosis of laryngopharyngeal reflux among globus patients. Otolaryngol Head Neck Surg. 2006; 134: 81-85. 